\title{
Well-posedness and computation of solutions of a regularized Benjamin-Ono system
}

\author{
Felipe Alexander Pipicano, Juan Carlos Muñoz Grajales* \\ Universidad del Valle, Departamento de Matemáticas, Cali, Colombia.
}

\begin{abstract}
This article is concerned with the existence and uniqueness of solutions of the Cauchy problem in the periodic setting for a regularized Benjamin-Ono type system (rBO) by using semigroup theory, Fourier analysis and Banach's fixed point theorem. This system was recently derived by Muñoz [12] as a weakly dispersive model for the propagation of small amplitude internal waves at the interface of two immiscible fluids with constant densities. We also conduct some numerical experiments to analyze the error and convergence in time and space of a fully discrete Fourier spectral scheme, for approximating the solutions of the initial value problem associated to the rBO system.
\end{abstract}

Keywords: Regularized BO system, internal waves, periodic travelling wave solutions, spectral methods.

MSC2010: 42A45, 47A75, 35Q51, 76M45.

\section{El buen planteamiento y el cálculo de soluciones de un sistema regularizado de Benjamin-Ono}

Resumen. En este artículo se estudia la existencia y la unicidad de soluciones del problema de Cauchy, en el caso periódico, para un sistema de tipo Benjamin-Ono regularizado (rBO), usando teoría de semigrupos, análisis de Fourier y el Teorema del punto fijo de Banach. Este sistema fue deducido recientemente por Muñoz [12] como un modelo débilmente dispersivo para la propagación de ondas internas con pequeña amplitud en la interface de dos fluidos inmiscibles con densidades constantes. Además se realizan algunos experimentos numéricos para analizar el error y la convergencia en tiempo y espacio de un esquema espectral de Fourier completamente discreto, a fin de aproximar las soluciones del problema de valor inicial asociado con el sistema rBO.

Palabras clave: Sistema BO regularizado, ondas internas, soluciones de onda viajera periódicas, métodos espectrales.

\footnotetext{
*E-mail: juan.munoz@correounivalle.edu.co

Received: 15 October 2015, Accepted: 05 February 2016

To cite this article: F.A. Pipicano, J.C. Muñoz Grajales, Well-posedness and computation of solutions of a regularized Benjamin-Ono system, Rev. Integr. Temas Mat. 34 (2016), No. 1, 59-80.
} 


\section{Introduction}

In this paper we will consider the nonlinear integro-differential system written in dimensionless variables

$$
\begin{aligned}
& \zeta_{t}-((1-\alpha \zeta) u)_{x}=\frac{\epsilon^{2}}{6} \zeta_{x x t}, \\
& u_{t}+\alpha u u_{x}-\rho \zeta_{x}=\frac{\rho_{2}}{\rho_{1}} \epsilon \mathcal{H}\left(u_{x t}\right)+\frac{\epsilon^{2}}{6} u_{x x t},
\end{aligned}
$$

under the initial conditions

$$
\zeta(x, 0)=\zeta_{0}(x) \quad \text { and } \quad u(x, 0)=u_{0}(x),
$$

and periodic boundary conditions

$$
\zeta(x, t)=\zeta(x+L, t) \quad \text { and } \quad u(x, t)=u(x+L, t),
$$

where $L>0$ is a positive constant. The symbol $\mathcal{H}$ denotes the periodic Hilbert Transform defined by

$$
\mathcal{H} f(x)=\frac{1}{L} \text { p.v. } \int_{-L / 2}^{L / 2} \cot \left(\frac{\pi(\xi-x)}{L}\right) f(\xi) d \xi,
$$

where the expression p.v. $\int$ stands for the integration in the principal value sense. We refer the reader to the works by Duoandikoetxea [8] and Butzer and Nesser [3] for more information about the Hilbert Transform. In particular, the following property of this linear operator is important in the present paper:

$$
\mathcal{H}\left(e^{i k x}\right)=i \operatorname{sign}(k) e^{i k x}, \quad k \in \mathbb{Z},
$$

where

$$
\operatorname{sign}(k)= \begin{cases}-1, & k<0, \\ 0, & k=0 \\ 1, & k>0 .\end{cases}
$$

The system above was deduced by Muñoz [12] and it describes the propagation of a weakly nonlinear $(\alpha<<1)$ internal wave propagating at the interface of two immiscible fluids with constant densities, which are contained at rest in a long channel $(\epsilon<<1)$ with a horizontal rigid top and bottom, and the thickness of the lower layer is assumed to be effectively infinite, i.e., $h_{2}>>h_{1}$ (deep water limit). In Figure 1 , we sketch the physical setting of the problem. In system (1), the constant $\rho$ is given by $\frac{\rho_{2}}{\rho_{1}}-1$, where $\rho_{1}$ and $\rho_{2}$ represent the densities of the fluids and $\rho_{2} / \rho_{1}>1$ (for stable stratification). The constants $\alpha$ and $\epsilon$ are small positive real numbers that measure the intensity of nonlinear and dispersive effects, respectively, $\alpha=\frac{a}{h_{1}}$ and $\epsilon=\frac{h_{1}}{L}$, where $h_{1}$ denotes the thickness of the upper fluid layer and the parameters $L$ and $a$ correspond to the characteristic wavelength and characteristic wave amplitude, respectively. The dimensionless variable $x$ represents the spatial position and $t$ the propagation time. The function $u=u(x, t)$ is the velocity monitored at the normalized depth $z=1-\sqrt{2 / 3}$, and $\zeta=\zeta(x, t)$ is the wave amplitude at the point $x$ and time $t$, measured with respect to the rest level of the twofluid interface. The phenomenon of propagation of waves on the interface between two 
layers of immiscible fluids of different densities is attracting the interest of many physicians and mathematicians, for both well-posedness theory and asymptotic theory, due to the challenging modelling and mathematical and numerical difficulties involved in the analysis of this physical system. Previous mathematical models for describing physically different scaling regimes of this phenomenon are those by Choi and Camassa [5], [6], [7]. On the other hand, Bona et al. [2] proposed a general method to derive systematically different weakly-dispersive, weakly nonlinear asymptotic models for the propagation of internal waves at a two-fluid interface. The system of variables used in [2] further enable the establishment of analytical results on consistency and convergence of the approximate models to the full Euler equations for an ideal, incompressible, irrotational fluid.

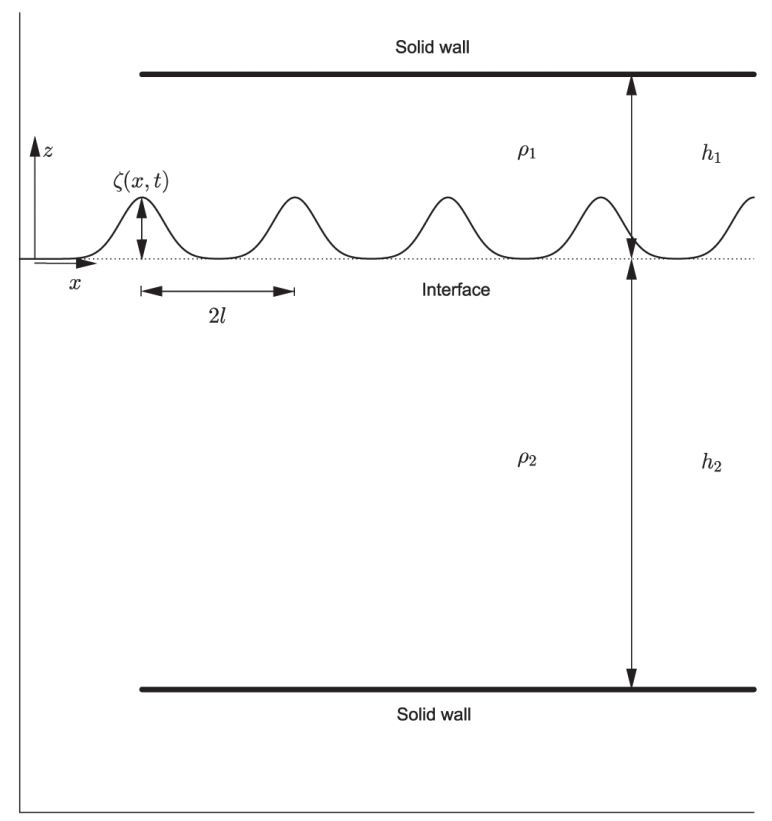

Figure 1. A typical periodic internal wave propagating at the interface of the two-fluid system which are contained at rest in a long channel with a horizontal rigid top and bottom.

In [12], Muñoz also established the existence and uniqueness of solutions in the nonperiodic case, and formulated a spectral scheme for approximating solutions of the corresponding Cauchy problem. Pipicano and Muñoz in [14] studied the existence of periodic travelling wave solutions of system (1) by using the topological-degree theory of positive operators on Banach spaces earlier developed in the works by Krasnosel'skii and Granas [10], [11], [9]. The technique used in [14] was inspired by Benjamin et al. [1] on solitary wave solutions of the Korteweg-de Vries (KdV) equation and Chen [4] in the framework of periodic travelling wave solutions. Quintero and Muñoz [15] also studied existence of non periodic travelling waves (solitary waves) of system (1) by adapting the theory on positive operators on cones applied in [1] to the KdV equation.

In order to continue the study on the properties of system (1), the first objective of the

Vol. 34, No. 1, 2016] 
present paper is to show that system (1) is locally well posed in the product periodic Sobolev space $H_{\text {per }}^{s} \times H_{\text {per }}^{s}$, provided that $s \geq 0$, by using semigroup theory and the Banach fixed point principle. This theory can be used to solve a wide class of problems commonly known as evolution equations that arise in many areas of application such as physics, chemistry, biology, engineering and economics, among others. Existence and uniqueness of system (1) is necessary in order to study, in a future research, the stability of travelling wave solutions of system (1).

The second purpose of this paper is to conduct some numerical experiments to analyze the accuracy of the fully discrete solver, introduced by Muñoz in [12], for approximating solutions of system (1) in both the linear and nonlinear cases on a spatial periodic domain. In [12] was only considered the error of the corresponding semi-discrete formulation. It is important to notice that the presence of the nonlocal dispersive operator $\mathcal{H}$ in system (1) makes the numerical investigation harder than the study of equations with only local terms. In this numerical method, the system is discretized in space by the Fourier spectral method and in time by a second-order accurate scheme. A potential application of this numerical tool is to explore the admissible range of velocity to guarantee the existence of solitary waves of the system and to establish whether they are orbitally stable/unstable under small disturbances.

The paper is organized as follows. In Section 2, we introduce notation, definitions and results necessary for the theory developed in the paper. In Section 3, we establish the local well-posedness of system (1) in the periodic case by using semigroup theory and Banach's fixed point theorem. Finally in Section 4, we present the numerical results obtained with a scheme which uses spectral discretization in space and a second-order finite difference approximation for time stepping of the initial value problem associated to system (1).

\section{Preliminaries}

Throughout this paper we will work with $L$-periodic functions, where $L$ is a positive real number. The symbol $\mathcal{K}$ will denote a positive constant that is updated according to the context.

Let $\mathbb{N}, \mathbb{Z}, \mathbb{R}$ and $\mathbb{C}$ be the sets of naturals, integers, reals and complex numbers, respectively. We will denote by $L^{2}(0, L)=L^{2}$ the Banach space of the all Lebesgue-measurable functions on $\mathbb{C}$ which are 2-integrable on $[0, L]$. The usual norm defined on $L^{2}$ is

$$
\|f\|_{L^{2}}:=\left(\int_{0}^{L}|f(x)|^{2} d x\right)^{1 / 2} .
$$

Additionally,

$$
\langle f, g\rangle=\int_{0}^{L} f(x) \overline{g(x)} d x
$$

is an inner product in $L^{2}$ and $\|f\|_{L^{2}}=\langle f, f\rangle^{1 / 2}$.

Let $C_{p e r}^{k}(0, L)=C_{p e r}^{k}, k=0,1,2, \ldots$ be the space of all $k$ times continuously-differentiable functions (or class $C^{k}$ ) of period $L$. Further, $C_{p e r}=C_{p e r}(0, L)=C_{p e r}^{0}(0, L)$ is the space 
of all continuous functions that are $L$-periodic and $C_{p e r}^{\infty}=C_{p e r}^{\infty}(0, L)=\bigcap_{k} C_{p e r}^{k}$. We will denote by $\mathcal{P}$ the space of all functions $\phi: \mathbb{R} \rightarrow \mathbb{C}$ of class $C^{\infty}$, that are $L$-periodic. We say that $T: \mathcal{P} \rightarrow \mathbb{C}$ defines a periodic distribution, i.e., $T \in \mathcal{P}^{\prime}$, if $T$ is linear and there exist a sequence $\left(\Psi_{n}\right)_{n \in \mathbb{N}} \subset \mathcal{P}$ such that

$$
T(\phi)=\lim _{n \rightarrow \infty} \int_{0}^{L} \Psi_{n}(x) \phi(x) d x, \quad \forall \phi \in \mathcal{P} .
$$

Let $s \in \mathbb{R}$. The Sobolev space, denoted by $H_{\text {per }}^{s}=H_{\text {per }}^{s}(0, L)$, is defined as

$$
H_{\text {per }}^{s}=\left\{f \in \mathcal{P}^{\prime}:\|f\|_{s}^{2}:=\sum_{n=-\infty}^{\infty}\left(1+n^{2}\right)^{s}|\widehat{f}(n)|^{2}<\infty\right\},
$$

where $\widehat{f}: \mathbb{Z} \rightarrow \mathbb{C}$ represents the coefficient of the Fourier Transform of $f$ define by

$$
\widehat{f}(n)=\frac{1}{L}\left\langle f, e^{-2 \pi i n x / L}\right\rangle .
$$

If $f \in C_{p e r}, \widehat{f}(n)$ can be written as

$$
\widehat{f}(n)=\frac{1}{L} \int_{0}^{L} f(x) e^{-2 \pi i n x / L} d x, \quad n \in \mathbb{Z} .
$$

We recall that for $s>1 / 2$, the space $H_{\text {per }}^{s}$ is an algebra, i.e., $\|f g\|_{s} \leq \mathcal{K}\|f\|_{s}\|g\|_{s}$.

We will denote by $\mathcal{X}_{s}$ the product space $H_{p e r}^{s} \times H_{p e r}^{s}$. It is easy to show that the expression

$$
\|Y\|_{\mathcal{X}_{s}}=\left(\|\zeta\|_{s}^{2}+\|u\|_{s}^{2}\right)^{1 / 2}
$$

defines a norm in $\mathcal{X}_{s}$ with $Y=(\zeta, u)^{T}$. Sometimes we will also use the equivalent norm

$$
\|Y\|_{\mathcal{X}_{s}}=\|\zeta\|_{s}+\|u\|_{s} .
$$

Finally, we recall some definitions about semigroup theory. Let $X$ be a Banach space. A one parameter family $(\mathcal{T}(t))_{t \geq 0}$ of bounded linear operators from $X$ into $X$ is a semigroup of bounded linear operators on $X$ if

i. $\mathcal{T}(0)=I,(I$ is the identity operator on $X)$;

ii. $\mathcal{T}(t+s)=\mathcal{T}(t) \mathcal{T}(s), \quad \forall t, s \geq 0$ (the semigroup property).

The linear operator $\mathcal{A}$ defined by

$$
\mathcal{A} x=\lim _{t \rightarrow 0^{+}} \frac{\mathcal{T}(t) x-x}{t} \quad \forall x \in D(\mathcal{A}),
$$

with domain

$$
D(\mathcal{A})=\left\{x \in X: \lim _{t \rightarrow 0^{+}} \frac{\mathcal{T}(t) x-x}{t} \text { exists }\right\},
$$

Vol. 34, No. 1, 2016] 
is called the infinitesimal generator of the semigroup $\mathcal{T}(t)$. A semigroup $(\mathcal{T}(t))_{t \geq 0}$ of bounded linear operators on $X$ is called a $C_{0}$ semigroup if

$$
\lim _{t \rightarrow 0^{+}} \mathcal{T}(t) x=x \quad \forall x \in X .
$$

If $C_{0}$ is a semigroup, $D(\mathcal{A})$ is dense on $X$. We refer the reader to [13] for a detailed information about semigroups of bounded linear operators.

\section{Existence and uniqueness}

In this section we will investigate the local well-posedness for the regularized BenjaminOno system

$$
\begin{aligned}
& \zeta_{t}-((1-\alpha \zeta) u)_{x}=\frac{\epsilon^{2}}{6} \zeta_{x x t}, \\
& u_{t}+\alpha u u_{x}-\rho \zeta_{x}=\frac{\rho_{2}}{\rho_{1}} \epsilon \mathcal{H}\left(u_{x t}\right)+\frac{\epsilon^{2}}{6} u_{x x t},
\end{aligned}
$$

where $\rho=\frac{\rho_{2}}{\rho_{1}}-1$, under the initial conditions

$$
\zeta(x, 0)=\zeta_{0}(x) \quad \text { and } \quad u(x, 0)=u_{0}(x),
$$

and periodic boundary conditions

$$
\zeta(x, t)=\zeta(x+L, t) \quad \text { and } \quad u(x, t)=u(x+L, t) .
$$

The system in (3) can be written as

$$
\left(\begin{array}{l}
\zeta_{t} \\
u_{t}
\end{array}\right)=\left(\begin{array}{cc}
0 & A^{-1} D \\
\rho B^{-1} D & 0
\end{array}\right)\left(\begin{array}{l}
\zeta \\
u
\end{array}\right)+\left(\begin{array}{cc}
0 & A^{-1} D \\
\rho B^{-1} D & 0
\end{array}\right)\left(\begin{array}{c}
-\frac{\alpha}{2 \rho} u^{2} \\
-\alpha \zeta u
\end{array}\right)
$$

where

$$
D=\partial_{x}, \quad A=\left(I-\frac{\epsilon^{2}}{6} \partial_{x x}\right) \quad \text { and } \quad B=\left(I-\frac{\epsilon^{2}}{6} \partial_{x x}-\frac{\rho_{2}}{\rho_{1}} \epsilon \mathcal{H} \partial_{x}\right) .
$$

If we denote

$$
Y(t)=\left(\begin{array}{l}
\zeta(t) \\
u(t)
\end{array}\right), \quad \mathcal{A}=\left(\begin{array}{cc}
0 & A^{-1} D \\
\rho B^{-1} D & 0
\end{array}\right) \quad \text { and } \quad F(Y)=\left(\begin{array}{c}
-\frac{\alpha}{2 \rho} u^{2} \\
-\alpha \zeta u
\end{array}\right),
$$

we conclude that $Y$ formally must satisfy the following initial value problem:

$$
\left\{\begin{array}{l}
\partial_{t} Y=\mathcal{A}(Y)+\mathcal{A} F(Y), \\
Y(0)=Y_{0}=\left(\begin{array}{c}
\zeta_{0} \\
u_{0}
\end{array}\right) .
\end{array}\right.
$$

Remark 3.1. The operators $A, B: H_{\text {per }}^{s} \rightarrow H_{\text {per }}^{s-2}$ are linear and bounded. In fact, let $\phi \in H_{\text {per }}^{s}$ and let us denote $w_{n}=\frac{2 \pi n}{L}$. Observe that

$$
\|A \phi\|_{s-2}^{2}=\sum_{n}\left(1+n^{2}\right)^{s-2}\left[1+\frac{\epsilon^{2} w_{n}^{2}}{6}\right]^{2}\left|\phi_{n}\right|^{2} \leq \mathcal{K} \sum_{n}\left(1+n^{2}\right)^{s}\left|\phi_{n}\right|^{2}=\mathcal{K}\|\phi\|_{s}^{2}
$$


and

$$
\|B \phi\|_{s-2}^{2}=\sum_{n}\left(1+n^{2}\right)^{s-2}\left[1+\frac{\epsilon^{2} w_{n}^{2}}{6}+\frac{\rho_{2} \epsilon\left|w_{n}\right|}{\rho_{1}}\right]^{2}\left|\phi_{n}\right|^{2} \leq \mathcal{K} \sum_{n}\left(1+n^{2}\right)^{s}\left|\phi_{n}\right|^{2}=\mathcal{K}\|\phi\|_{s}^{2} .
$$

Let us define the operators $A^{-1}, B^{-1}: H_{\text {per }}^{s-2} \rightarrow H_{\text {per }}^{s}$ as

$$
A^{-1} \phi=\sum_{n} \frac{\phi_{n}}{1+\frac{\epsilon^{2} w_{n}^{2}}{6}} e^{i w_{n} x} \quad \text { and } \quad B^{-1} \phi=\sum_{n} \frac{\phi_{n}}{1+\frac{\epsilon^{2} w_{n}^{2}}{6}+\frac{\rho_{2} \epsilon\left|w_{n}\right|}{\rho_{1}}} e^{i w_{n} x} .
$$

Note that the operators $A^{-1}$ and $B^{-1}$ are also linear and bounded. It follows that the operator $\mathcal{A}: \mathcal{X}_{s-1} \rightarrow \mathcal{X}_{s}$ is linear and bounded. In fact, the explicit form of $\mathcal{A}$ is

$$
\mathcal{A}\left(\begin{array}{l}
\zeta \\
u
\end{array}\right)=\sum_{n \neq 0}\left(\begin{array}{c}
i \sigma_{n} u_{n} \\
i \gamma_{n} \zeta_{n}
\end{array}\right) e^{i w_{n} x}
$$

where

$$
\sigma_{n}=\frac{w_{n}}{1+\frac{\epsilon^{2} w_{n}^{2}}{6}} \quad \text { and } \quad \gamma_{n}=\frac{\rho w_{n}}{1+\frac{\epsilon^{2} w_{n}^{2}}{6}+\frac{\rho_{2} \epsilon\left|w_{n}\right|}{\rho_{1}}} .
$$

For our purposes, in this article, we consider the operator $\mathcal{A}$ from $\mathcal{X}_{s}$ into $\mathcal{X}_{s}, s \geq 0$.

\subsection{Linear problem}

In this part, we consider the linear case of system (4):

$$
\left\{\begin{array}{l}
\partial_{t} Y=\mathcal{A}(Y), \\
Y(0)=Y_{0}
\end{array}\right.
$$

To construct the linear semigroup of operators, for $s \geq 0$, we consider the expansions

$$
\zeta(x, t)=\sum_{n} \zeta_{n}(t) e^{i w_{n} x} \quad \text { and } \quad u(x, t)=\sum_{n} u_{n}(t) e^{i w_{n} x} .
$$

By replacing the expressions of (6) into (5) we obtain

$$
\left\{\begin{array}{l}
\sum_{n} \zeta_{n}^{\prime}(t) e^{i w_{n} x}=\sum_{n} u_{n}(t) i \sigma_{n} e^{i w_{n} x}, \\
\sum_{n}^{n} u_{n}^{\prime}(t) e^{i w_{n} x}=\sum_{n} \zeta_{n}(t) i \gamma_{n} e^{i w_{n} x} .
\end{array}\right.
$$

Therefore, for each $n \in \mathbb{Z}$ we have the system

$$
\left\{\begin{array}{l}
\zeta_{n}^{\prime}(t)=i \sigma_{n} u_{n}(t), \\
u_{n}^{\prime}(t)=i \gamma_{n} \zeta_{n}(t),
\end{array}\right.
$$

under the initial conditions

$$
\zeta_{n}(0)=\zeta_{0, n} \quad \text { and } \quad u_{n}(0)=u_{0, n} .
$$

Vol. 34, No. 1, 2016] 
A direct calculation shows that the explicit solutions of this system are given by

$$
\left\{\begin{array}{l}
\zeta_{n}(t)=\zeta_{0, n} \cos \left(\sqrt{\sigma_{n} \gamma_{n}} t\right)+i \Gamma_{n} u_{0, n} \sin \left(\sqrt{\sigma_{n} \gamma_{n}} t\right), \\
u_{n}(t)=u_{0, n} \cos \left(\sqrt{\sigma_{n} \gamma_{n}} t\right)+i \Theta_{n} \zeta_{0, n} \sin \left(\sqrt{\sigma_{n} \gamma_{n}} t\right),
\end{array}\right.
$$

for $n \neq 0$, and

$$
\zeta_{0}(t)=\zeta_{0,0} \quad \text { and } \quad u_{0}(t)=u_{0,0}
$$

Here

$$
\Gamma_{n}=\operatorname{sign}\left(\gamma_{n}\right) \sqrt{\frac{\sigma_{n}}{\gamma_{n}}}, \quad \Theta_{n}=\frac{1}{\Gamma_{n}}, \quad n \neq 0,
$$

$\Gamma_{0}=\Theta_{0}=0$, where $\operatorname{sign}(x)$ denotes the sign function of $x$. Here we used the property of the Hilbert Transform given in (2).

Let us define for $t \geq 0$ and

$$
Y=\left(\begin{array}{l}
\zeta \\
u
\end{array}\right)=\sum_{n}\left(\begin{array}{c}
\zeta_{n}(t) \\
u_{n}(t)
\end{array}\right) e^{i w_{n} x}
$$

the family of linear operators

$$
\mathcal{T}(t)(Y)=\sum_{n \neq 0} \mathcal{M}_{n}(t)\left(\begin{array}{c}
\zeta_{n} \\
u_{n}
\end{array}\right) e^{i w_{n} x}+\left(\begin{array}{c}
\zeta_{0} \\
u_{0}
\end{array}\right),
$$

where

$$
\mathcal{M}_{n}(t)=\left(\begin{array}{cc}
\cos \left(\sqrt{\sigma_{n} \gamma_{n}} t\right) & i \Gamma_{n} \sin \left(\sqrt{\sigma_{n} \gamma_{n}} t\right) \\
i \Theta_{n} \sin \left(\sqrt{\sigma_{n} \gamma_{n}} t\right) & \cos \left(\sqrt{\sigma_{n} \gamma_{n}} t\right)
\end{array}\right)
$$

Note that if $Y=\left(\begin{array}{l}\zeta \\ u\end{array}\right)$ is an element of $\mathcal{X}_{s}, s \geq 0$, then

$$
\begin{aligned}
& \|\mathcal{T}(t) Y\|_{\mathcal{X}_{s}}^{2} \\
& \quad=\sum_{n \neq 0}\left(1+n^{2}\right)^{s}\left|\zeta_{n} \cos \left(\sqrt{\sigma_{n} \gamma_{n}} t\right)+i \Gamma_{n} u_{n} \sin \left(\sqrt{\sigma_{n} \gamma_{n}} t\right)\right|^{2}+\left|\zeta_{0}\right|^{2} \\
& \quad+\sum_{n \neq 0}\left(1+n^{2}\right)^{s}\left|u_{n} \cos \left(\sqrt{\sigma_{n} \gamma_{n}} t\right)+i \Theta_{n} \zeta_{n} \sin \left(\sqrt{\sigma_{n} \gamma_{n}} t\right)\right|^{2}+\left|u_{0}\right|^{2} \\
& \quad \leq \sum_{n \neq 0}\left(1+n^{2}\right)^{s} \mathcal{K}\left(\left|\zeta_{n}\right|^{2}+\left|u_{n}\right|^{2}\right)+\left|\zeta_{0}\right|^{2}+\sum_{n \neq 0}\left(1+n^{2}\right)^{s} \mathcal{K}\left(\left|\zeta_{n}\right|^{2}+\left|u_{n}\right|^{2}\right)+\left|u_{0}\right|^{2} \\
& \quad \leq \mathcal{K}\left(\|\zeta\|_{s}^{2}+\|\zeta\|_{s}^{2}\right)=\mathcal{K}\|Y\|_{\mathcal{X}_{s}}^{2},
\end{aligned}
$$

since

$$
\begin{aligned}
& \left|\zeta_{n} \cos \left(\sqrt{\sigma_{n} \gamma_{n}} t\right)+i \Gamma_{n} u_{n} \sin \left(\sqrt{\sigma_{n} \gamma_{n}} t\right)\right|^{2} \leq \mathcal{K}\left(\left|\zeta_{n}\right|^{2}+\left|u_{n}\right|^{2}\right), \\
& \left|u_{n} \cos \left(\sqrt{\sigma_{n} \gamma_{n}} t\right)+i \Theta_{n} \zeta_{n} \sin \left(\sqrt{\sigma_{n} \gamma_{n}} t\right)\right|^{2} \leq \mathcal{K}\left(\left|\zeta_{n}\right|^{2}+\left|u_{n}\right|^{2}\right),
\end{aligned}
$$

and due to the terms $\Gamma_{n}$ and $\Theta_{n}$ are controlled by a constant. Thus,

$$
\|\mathcal{T}(t) Y\|_{\mathcal{X}_{s}} \leq \mathcal{K}\|Y\|_{\mathcal{X}_{s}} .
$$

Therefore $(\mathcal{T}(t))_{t \geq 0}$ is well defined from $\mathcal{X}_{s}$ into $\mathcal{X}_{s}, s \geq 0$. 
Theorem 3.2. The family $(\mathcal{T}(t))_{t \geq 0}$ is a $C_{0}$-semigroup of linear and bounded operators in $\mathcal{X}_{s}$.

Proof. A direct calculation shows that $(\mathcal{T}(t))_{t>0}$ is a family of linear operators and each $\mathcal{T}(t)$ is bounded due to $(9)$.

Note that

$$
\mathcal{T}(0)(Y)=\sum_{n \neq 0}\left(\begin{array}{ll}
1 & 0 \\
0 & 1
\end{array}\right)\left(\begin{array}{l}
\zeta_{n} \\
u_{n}
\end{array}\right)+\left(\begin{array}{l}
\zeta_{0} \\
u_{0}
\end{array}\right)=Y .
$$

Thus, $\mathcal{T}(0)=I$. The semigroup property $\mathcal{T}(t+s)=\mathcal{T}(t) \mathcal{T}(s)$, is a consequence of the equality:

$$
\mathcal{M}_{n}(t+s)=\mathcal{M}_{n}(t) \mathcal{M}_{n}(s) .
$$

Hence, $(\mathcal{T}(t))_{t \geq 0}$ is a semigroup of linear and bounded operators on $\mathcal{X}_{s}$.

On the other hand, let $Y=\left(\begin{array}{l}\zeta \\ u\end{array}\right)$ be an element in $\mathcal{X}_{s}$. Then

$$
\begin{aligned}
\|\mathcal{T}(t) Y-Y\|_{\mathcal{X}_{s}}^{2} & =\sum_{n \neq 0}\left(1+n^{2}\right)^{s}\left|\zeta_{n}\left(\cos \left(\sqrt{\sigma_{n} \gamma_{n}} t\right)-1\right)+i \Gamma_{n} u_{n} \sin \left(\sqrt{\sigma_{n} \gamma_{n}} t\right)\right|^{2} \\
& +\sum_{n \neq 0}\left(1+n^{2}\right)^{s}\left|u_{n}\left(\cos \left(\sqrt{\sigma_{n} \gamma_{n}} t\right)-1\right)+i \Theta_{n} \zeta_{n} \sin \left(\sqrt{\sigma_{n} \gamma_{n}} t\right)\right|^{2} .
\end{aligned}
$$

Note that the series at the right side above converge uniformly in $t \geq 0$ with upper bounds similar to (8). Thus, as a consequence of the dominated convergence theorem for sums, we have

$$
\begin{aligned}
\lim _{t \rightarrow 0^{+}} & \|\mathcal{T}(t) Y-Y\|_{\mathcal{X}_{s}}^{2} \\
& =\sum_{n \neq 0} \lim _{t \rightarrow 0^{+}}\left(1+n^{2}\right)^{s}\left|\zeta_{n}\left(\cos \left(\sqrt{\sigma_{n} \gamma_{n}} t\right)-1\right)+i \Gamma_{n} u_{n} \sin \left(\sqrt{\sigma_{n} \gamma_{n}} t\right)\right|^{2} \\
& +\sum_{n \neq 0} \lim _{t \rightarrow 0^{+}}\left(1+n^{2}\right)^{s}\left|u_{n}\left(\cos \left(\sqrt{\sigma_{n} \gamma_{n}} t\right)-1\right)+i \Theta_{n} \zeta_{n} \sin \left(\sqrt{\sigma_{n} \gamma_{n}} t\right)\right|^{2} \\
& =0+0=0 .
\end{aligned}
$$

Thus $\lim _{t \rightarrow 0^{+}} \mathcal{T}(t) Y=Y$. This shows that $(\mathcal{T}(t))_{t \geq 0}$ is a $C_{0^{-}}$-semigroup.

Theorem 3.3. The linear operator $\mathcal{A}: \mathcal{X}_{s} \rightarrow \mathcal{X}_{s}$, with $s \geq 0$, is the infinitesimal generator of the semigroup $(\mathcal{T}(t))_{t \geq 0}$.

Proof. Note that

$$
\begin{aligned}
& \left\|\frac{1}{t}[\mathcal{T}(t)(Y)-Y]-\mathcal{A}(Y)\right\|_{\mathcal{X}_{s}} \\
& =\left\|\sum_{n \neq 0}\left(\begin{array}{l}
\frac{\cos \left(\sqrt{\sigma_{n} \gamma_{n}} t\right)-1}{t} \zeta_{n}+i \Gamma_{n} \frac{\sin \left(\sqrt{\sigma_{n} \gamma_{n}} t\right)}{t} u_{n}-i \sigma_{n} u_{n} \\
\frac{\cos \left(\sqrt{\sigma_{n} \gamma_{n}} t\right)-1}{t} u_{n}+i \Theta_{n} \frac{\sin \left(\sqrt{\sigma_{n} \gamma_{n}} t\right)}{t} \zeta_{n}-i \gamma_{n} \zeta_{n}
\end{array}\right)\right\|_{\mathcal{X}_{s}} .
\end{aligned}
$$

Vol. 34, No. 1, 2016] 
Note that the terms

$$
\frac{\cos \left(\sqrt{\sigma_{n} \gamma_{n}} t\right)-1}{t}, \quad \frac{\sin \left(\sqrt{\sigma_{n} \gamma_{n}} t\right)}{t}, \quad \Gamma_{n}, \quad \Theta_{n}, \quad \text { and } \quad \sqrt{\sigma_{n} \gamma_{n}}
$$

are controlled by a constant. This implies that

$$
\left|\frac{\cos \left(\sqrt{\sigma_{n} \gamma_{n}} t\right)-1}{t} \zeta_{n}+i \Gamma_{n} \frac{\sin \left(\sqrt{\sigma_{n} \gamma_{n}} t\right)}{t} u_{n}-i \sigma_{n} u_{n}\right|^{2} \leq \mathcal{K}\left|\zeta_{n}\right|^{2}
$$

and

$$
\left|\frac{\cos \left(\sqrt{\sigma_{n} \gamma_{n}} t\right)-1}{t} u_{n}+i \Theta_{n} \frac{\sin \left(\sqrt{\sigma_{n} \gamma_{n}} t\right)}{t} \zeta_{n}-i \gamma_{n} \zeta_{n}\right|^{2} \leq \mathcal{K}\left|u_{n}\right|^{2} .
$$

Therefore, the dominated convergence theorem for sums implies that

$$
\begin{aligned}
\lim _{t \rightarrow 0^{+}}\left\|\frac{1}{t}[\mathcal{T}(t)(Y)-Y]-\mathcal{A}(Y)\right\|_{\mathcal{X}_{s}} \\
\quad=\left\|\sum_{n \neq 0}\left(\begin{array}{l}
\lim _{t \rightarrow 0^{+}} \frac{\cos \left(\sqrt{\sigma_{n} \gamma_{n}} t\right)-1}{\cos \left(\sqrt{\sigma_{n} \gamma_{n}} t\right)-1} \zeta_{n}+i \Gamma_{n} \frac{\sin \left(\sqrt{\sigma_{n} \gamma_{n}} t\right)}{t} u_{n}+i \Theta_{n} \frac{\sin \left(\sqrt[t]{\sigma_{n} \gamma_{n}} t\right)}{t} \zeta_{n}-i \sigma_{n} u_{n} \\
\lim _{t \rightarrow 0^{+}} \frac{\operatorname{lo} \zeta_{n}}{t}
\end{array}\right)\right\|_{\mathcal{X}_{s}} \\
\quad=0 .
\end{aligned}
$$

Therefore $\mathcal{A}(Y)=\lim _{t \rightarrow 0^{+}}\left(\frac{\mathcal{T}(t)-I}{t}\right)(Y)$ for any $Y \in \mathcal{X}_{s}$, which proves that the operator $\mathcal{A}$ is the infinitesimal generator of the semigroup $(\mathcal{T}(t))_{t \geq 0}$.

\subsection{Nonlinear problem}

In this part, we study the existence, uniqueness and behavior of the solutions for the problem (4), under changes of the initial data.

Lemma 3.4. Let $\mathcal{L}_{1}=A^{-1} D$ and $\mathcal{L}_{2}=B^{-1} D, u \in H_{\text {per }}^{r}$ and $v \in H_{\text {per }}^{r^{\prime}}$ with $0 \leq r \leq s$, $0 \leq r^{\prime} \leq s, 0 \leq 2 s-r-r^{\prime}<1 / 4$. Then,

$$
\left\|\mathcal{L}_{1}(u v)\right\|_{H_{p e r}^{s}} \leq C_{r, r^{\prime}, s}\|u\|_{H_{p e r}^{r}}\|v\|_{H_{p e r}^{r^{\prime}}}
$$

and

$$
\left\|\mathcal{L}_{2}(u v)\right\|_{H_{p e r}^{s}} \leq C_{r, r^{\prime}, s}\|u\|_{H_{p e r}^{r}}\|v\|_{H_{p e r}^{r^{\prime}}}
$$

where $C_{r, r^{\prime}, s}$ is a constant depending on $r, r^{\prime}, s$.

Proof. Let us observe that

$$
\begin{gathered}
\widehat{\mathcal{L}_{1} u}(k)=\frac{i w_{n}}{1+\frac{\epsilon^{2} w_{n}^{2}}{6}} \widehat{u}(k), \\
\widehat{\mathcal{L}_{2} u}(k)=\frac{i w_{n}}{1+\frac{\epsilon^{2} w_{n}^{2}}{6}+\frac{\rho_{2}}{\rho_{1}} \epsilon\left|w_{n}\right|} \widehat{u}(k),
\end{gathered}
$$


and

$$
\begin{gathered}
\frac{\left|i w_{n}\right|}{1+\frac{\epsilon^{2} w_{n}^{2}}{6}} \leq \frac{C|n|}{1+n^{2}}, \\
\frac{\left|i w_{n}\right|}{1+\frac{\epsilon^{2} w_{n}^{2}}{6}+\frac{\rho_{2}}{\rho_{1}} \epsilon\left|w_{n}\right|} \leq \frac{C|n|}{1+n^{2}} .
\end{gathered}
$$

Therefore, the proof is analogous to Lemma 3.1 in [16].

Lemma 3.5. If $Y, \bar{Y} \in C\left([0, T], \mathcal{X}_{s}\right)$ with $s \geq 0$, then, for $Y=(\zeta, u)^{T}$ and $\bar{Y}=(\bar{\zeta}, \bar{u})^{T}$ we have

$$
\|\mathcal{A}(F(Y)-F(\bar{Y}))\|_{\mathcal{X}_{s}} \leq\|Y-\bar{Y}\|_{\mathcal{X}_{s}} L\left(\|u\|_{s},\|\bar{u}\|_{s},\|\bar{\zeta}\|_{s}\right),
$$

where $L$ is a linear polynomial depending on $\|u\|_{s},\|\bar{u}\|_{s}$ and $\|\bar{\zeta}\|_{s}$.

Proof. First of all, suppose that $s>\frac{1}{2}$. Since $H_{\text {per }}^{s}$ is an algebra for $s>1 / 2$, we have

$$
\begin{aligned}
& \|\mathcal{A}(F(Y)-F(\bar{Y}))\|_{\mathcal{X}_{s}} \leq \mathcal{K}\|F(Y)-F(\bar{Y})\|_{\mathcal{X}_{s}} \\
& \quad=\mathcal{K}\left[\left\|u^{2}-\bar{u}^{2}\right\|_{s}+\|\zeta u-\bar{\zeta} \bar{u}\|_{s}\right] \\
& \quad \leq \mathcal{K}\left(\|u-\bar{u}\|_{s}\|u+\bar{u}\|_{s}+\|\zeta-\bar{\zeta}\|_{s}\|u+\bar{u}\|_{s}+\|\zeta\|_{s}\|u-\bar{u}\|_{s}+\|u\|_{s}\|\zeta-\bar{\zeta}\|_{s}\right) \\
& \quad \leq \mathcal{K}\left(\|u-\bar{u}\|_{s}\left[\|u\|_{s}+\|\bar{u}\|_{s}+\|\zeta\|_{s}\right]+\|\zeta-\bar{\zeta}\|_{s}\left[\|u\|_{s}+\|\bar{u}\|_{s}\right]\right) \\
& \quad \leq L\left(\|u\|_{s},\|\bar{u}\|_{s},\|\bar{\zeta}\|_{s}\right)\|Y-\bar{Y}\|_{\mathcal{X}_{s}},
\end{aligned}
$$

where $L\left(\|u\|_{s},\|\bar{u}\|_{s},\|\bar{\zeta}\|_{s}\right)=\mathcal{K}\left(\|u\|_{s}+\|\bar{u}\|_{s}+\|\zeta\|_{s}\right)$.

On the other hand, we recall that

$$
\mathcal{A}=\left(\begin{array}{cc}
0 & A^{-1} D \\
\rho B^{-1} D & 0
\end{array}\right)
$$

Thus, for $0 \leq s<1$, we can apply Lemma 3.4 with $r=\frac{3 s}{4}<s, r^{\prime}=s$, to get again the inequality (10).

Theorem 3.6. If $Y \in C\left([0, T] ; \mathcal{X}_{s}\right)$ with $s \geq 0$ is a solution of (4), then $Y$ satisfies the integral equation

$$
Y(t)=\mathcal{T}(t) Y_{0}+\int_{0}^{t} \mathcal{T}(t-\xi) \mathcal{A} F(Y(\xi)) d \xi
$$

Analogously, if $Y \in C\left([0, T] ; \mathcal{X}_{s}\right), s \geq 0$, is a solution of $(11)$, then $Y \in C^{1}\left([0, T] ; \mathcal{X}_{s}\right)$ and satisfies (4) in the following sense:

$$
\lim _{h \rightarrow 0^{+}}\left\|\frac{Y(t+h)-Y(t)}{h}-\mathcal{A}(Y(t))-\mathcal{A} F(Y(t))\right\|_{\mathcal{X}_{s}}=0 .
$$

Proof. Let $Y(t) \in C\left([0, T] ; \mathcal{X}_{s}\right)$ be a solution of the IVP (4). Then, for $0 \leq \xi \leq t$ we have

$$
\mathcal{T}(t-\xi) Y^{\prime}(\xi)=\mathcal{T}(t-\xi) \mathcal{A}(Y(\xi))+\mathcal{T}(t-\xi) \mathcal{A} F(Y(\xi)) .
$$


Note that

$$
\frac{d}{d \xi}(\mathcal{T}(t-\xi) Y(\xi))=\mathcal{T}(t-\xi) Y^{\prime}(\xi)-\mathcal{T}(t-\xi) \mathcal{A}(Y(\xi))
$$

Thus,

$$
\frac{d}{d \xi}(\mathcal{T}(t-\xi) Y(\xi))=\mathcal{T}(t-\xi) \mathcal{A} F(Y(\xi))
$$

Integrating on both sides of the equation (12), we obtain that $Y$ satisfies the integral equation

$$
Y(t)=\mathcal{T}(t) Y_{0}+\int_{0}^{t} \mathcal{T}(t-\xi) \mathcal{A} F(Y(\xi)) d \xi
$$

On the other hand, suppose that $Y(t) \in C\left([0, T] ; \mathcal{X}_{s}\right)$ is a solution of the integral equation (11). Consider the expression

$$
\Gamma:=\left\|\frac{Y(t+h)-Y(t)}{h}-\mathcal{A}(Y(t))-\mathcal{A} F(Y(t))\right\|_{\mathcal{X}_{s}} .
$$

Replacing the expression (11) into (13) and applying the triangle inequality, we obtain

$$
\begin{aligned}
\Gamma & \leq\left\|\mathcal{T}(t)\left(\frac{\mathcal{T}(h)-I}{h}\right) Y_{0}-\mathcal{T}(t) \mathcal{A}\left(Y_{0}\right)\right\|_{\mathcal{X}_{s}} \\
& +\left\|\left[\frac{\mathcal{T}(h)-I}{h}-\mathcal{A}\right] \int_{0}^{t} \mathcal{T}(t-\xi) \mathcal{A} F(Y(\xi)) d \xi\right\|_{\mathcal{X}_{s}} \| \\
& +\left\|\frac{1}{h} \int_{t}^{t+h} \mathcal{T}(t+h-\xi) \mathcal{A} F(Y(\xi)) d \xi-\mathcal{A} F(Y(t))\right\|_{\mathcal{X}_{s}} .
\end{aligned}
$$

Note that

$$
\begin{aligned}
& \lim _{h \rightarrow 0^{+}}\left\|\mathcal{T}(t)\left(\frac{\mathcal{T}(h)-I}{h}\right) Y_{0}-\mathcal{T}(t) \mathcal{A}\left(Y_{0}\right)\right\|_{\mathcal{X}_{s}}=0, \\
& \lim _{h \rightarrow 0^{+}}\left\|\left[\frac{\mathcal{T}(h)-I}{h}-\mathcal{A}\right] \int_{0}^{t} \mathcal{T}(t-\xi) \mathcal{A} F(Y(\xi)) d \xi\right\|_{\mathcal{X}_{s}}=0 .
\end{aligned}
$$

The last term can be controlled by using the mean value theorem in the following way:

$$
\begin{aligned}
& \left\|\frac{1}{h} \int_{t}^{t+h} \mathcal{T}(t+h-\xi) \mathcal{A} F(Y(\xi)) d \xi-\mathcal{A} F(Y(t))\right\|_{\mathcal{X}_{s}} \\
& \quad \leq \frac{1}{h} \int_{t}^{t+h}\|\mathcal{T}(t+h-\xi) \mathcal{A} F(Y(\xi))-\mathcal{A} F(Y(t))\|_{\mathcal{X}_{s}} d \xi \\
& \quad=\|\mathcal{T}(t+h-\tilde{\xi}) \mathcal{A} F(Y(\tilde{\xi}))-\mathcal{A} F(Y(t))\|_{\mathcal{X}_{s}} \\
& \quad \leq\|\mathcal{T}(t+h-\tilde{\xi}) \mathcal{A}[F(Y(\tilde{\xi}))-F(Y(t))]\|_{\mathcal{X}_{s}}+\|[\mathcal{T}(t+h-\tilde{\xi})-I] \mathcal{A} F(Y(t))\|_{\mathcal{X}_{s}},
\end{aligned}
$$


for some $t \leq \tilde{\xi} \leq t+h$. Observe that the last term in (15) satisfies

$$
\lim _{h \rightarrow 0^{+}}\|[\mathcal{T}(t+h-\tilde{\xi})-I] \mathcal{A} F(Y(t))\|_{\mathcal{X}_{s}}=0,
$$

since $h \rightarrow 0^{+}$implies $\tilde{\xi} \rightarrow 0^{+}$. Furthermore, by using Lemma 3.5, we have that the first term in (15) can be bounded as

$$
\begin{gathered}
\|\mathcal{T}(t+h-\tilde{\xi}) \mathcal{A}[F(Y(\tilde{\xi}))-F(Y(t))]\|_{\mathcal{X}_{s}} \leq \mathcal{K}\|\mathcal{A}[F(Y(\tilde{\xi}))-F(Y(t))]\|_{\mathcal{X}_{s}} \\
\leq L\left(\|u\|_{s}, 0,\|\zeta\|_{s}\right)\|F(Y(\tilde{\xi}))-F(Y(t))\|_{\mathcal{X}_{s}} \rightarrow 0,
\end{gathered}
$$

as $h \rightarrow 0^{+}$. Hence,

$$
\lim _{h \rightarrow 0^{+}}\left\|\frac{Y(t+h)-Y(t)}{h}-\mathcal{A}(Y(t))-\mathcal{A} F(Y(t))\right\|_{\mathcal{X}_{s}}=0 .
$$

Theorem 3.7. Let $Y_{0} \in \mathcal{X}_{s}, s \geq 0$. Then there exist $T^{*}>0$ and $Y \in C\left([0, T] ; \mathcal{X}_{s}\right)$ satisfying the integral equation (11).

Proof. Let us define the set

$$
\mathcal{S}_{M}=\left\{Y=\left(\begin{array}{l}
\zeta \\
u
\end{array}\right) \in C\left([0, T] ; \mathcal{X}_{s}\right): \sup _{t \in[0, T]}\left\|Y(t)-\mathcal{T}(t) Y_{0}\right\|_{\mathcal{X}_{s}} \leq M\right\},
$$

with the norm $\|Y\|_{C\left([0, T], \mathcal{X}_{s}\right)}=\sup _{t \in[0, T]}\left(\|\zeta(t)\|_{s}+\|u(t)\|_{s}\right)$, where $Y=(\zeta, u)^{T}$. Observe that endowed with this norm, $\mathcal{S}_{M}$ is a complete set in $C\left([0, T] ; \mathcal{X}_{s}\right)$ and $F$ is continuous in $\mathcal{S}_{M}$ as a consequence of Lemma 3.5.

For $Y \in \mathcal{S}_{M}$, we define the operator

$$
\Psi(Y(t))=\mathcal{T}(t) Y_{0}+\int_{0}^{t} \mathcal{T}(t-\xi) \mathcal{A} F(Y(\xi)) d \xi
$$

Observe that

$$
\begin{gathered}
\|\Psi(Y(t+h))-\Psi(Y(t))\|_{\mathcal{X}_{s}} \\
=\| \mathcal{T}(t+h) Y_{0}+\int_{0}^{t+h} \mathcal{T}(t+h-\xi) \mathcal{A} F(Y(\xi)) d \xi-\mathcal{T}(t) Y_{0} \\
\quad-\int_{0}^{t} \mathcal{T}(t-\xi) \mathcal{A} F(Y(\xi)) d \xi \|_{\mathcal{X}_{s}} \\
\leq\left\|(\mathcal{T}(t+h)-\mathcal{T}(t)) Y_{0}\right\|_{\mathcal{X}_{s}}+\left\|\int_{0}^{t}(\mathcal{T}(t+h-\xi)-\mathcal{T}(t-\xi)) \mathcal{A} F(Y(\xi)) d \xi\right\|_{\mathcal{X}_{s}} \\
\quad+\left\|\int_{t}^{t+h} \mathcal{T}(t+h-\xi) \mathcal{A} F(Y(\xi)) d \xi\right\|_{\mathcal{X}_{s}}
\end{gathered}
$$

Note that the first term at the right side above satisfies

$$
\lim _{h \rightarrow 0^{+}}\left\|(\mathcal{T}(t+h)-\mathcal{T}(t)) Y_{0}\right\|_{\mathcal{X}_{s}}=0,
$$

Vol. 34, No. 1, 2016] 
since $(\mathcal{T}(t))_{t \geq 0}$ is a $C_{0}$-semigroup in $\mathcal{X}_{s}$. The second and third terms at the right side are controlled using the Lebesgue's dominated convergence theorem. Note that

$$
\|(\mathcal{T}(t+h-\xi)-\mathcal{T}(t-\xi)) \mathcal{A} F(Y(\xi))\|_{\mathcal{X}_{s}} \leq \mathcal{K}\|\mathcal{A} F(Y(\xi))\|_{\mathcal{X}_{s}} \leq \mathcal{K}\|F(Y(\xi))\|_{\mathcal{X}_{s}},
$$

and

$$
\lim _{h \rightarrow 0^{+}}\|(\mathcal{T}(t+h-\xi)-\mathcal{T}(t-\xi)) \mathcal{A} F(Y(\xi))\|_{\mathcal{X}_{s}}=0
$$

Thus,

$$
\begin{aligned}
\lim _{h \rightarrow 0^{+}} & \left\|\int_{0}^{t}(\mathcal{T}(t+h-\xi)-\mathcal{T}(t-\xi)) \mathcal{A} F(Y(\xi)) d \xi\right\|_{\mathcal{X}_{s}} \\
& \leq \lim _{h \rightarrow 0^{+}} \int_{0}^{t}\|(\mathcal{T}(t+h-\xi)-\mathcal{T}(t-\xi)) \mathcal{A} F(Y(\xi))\|_{\mathcal{X}_{s}} d \xi=0 .
\end{aligned}
$$

Finally,

$$
\begin{aligned}
\|\mathcal{T}(t+h-\xi) \mathcal{A} F(Y(\xi))\|_{\mathcal{X}_{s}} & \leq L\left(\|u\|_{s}, 0,\|\zeta\|_{s}\right)\|Y(\xi)\|_{\mathcal{X}_{s}} \\
& =\mathcal{K}\left(\|u\|_{\mathcal{X}_{s}}+\|\zeta\|_{\mathcal{X}_{s}}\right)\left(\|u\|_{\mathcal{X}_{s}}+\|\zeta\|_{\mathcal{X}_{s}}\right) \\
& =\mathcal{K}\left(\|u\|_{\mathcal{X}_{s}}+\|\zeta\|_{\mathcal{X}_{s}}\right)^{2} \\
& =\mathcal{K}\|Y\|_{\mathcal{X}_{s}}^{2} \\
& \leq\left(M+\mathcal{K}\left\|Y_{0}\right\|_{\mathcal{X}_{s}}\right)^{2},
\end{aligned}
$$

which is a consequence of the estimate

$$
\begin{aligned}
\|Y(t)\|_{\mathcal{X}_{s}} & =\left\|Y(t)-\mathcal{T}(t) Y_{0}+\mathcal{T}(t) Y_{0}\right\|_{\mathcal{X}_{s}} \\
& \leq\left\|Y(t)-\mathcal{T}(t) Y_{0}\right\|_{\mathcal{X}_{s}}+\left\|\mathcal{T}(t) Y_{0}\right\|_{\mathcal{X}_{s}} \\
& \leq\left(M+\mathcal{K}\left\|Y_{0}\right\|_{\mathcal{X}_{s}}\right) .
\end{aligned}
$$

Since

$$
\lim _{h \rightarrow 0^{+}}\|\mathcal{T}(t+h-\xi) \mathcal{A} F(Y(\xi))\|_{\mathcal{X}_{s}}=0
$$

it follows that

$$
\begin{aligned}
\lim _{h \rightarrow 0^{+}}\left\|\int_{t}^{t+h} \mathcal{T}(t+h-\xi) \mathcal{A} F(Y(\xi)) d \xi\right\|_{\mathcal{X}_{s}} & \leq \lim _{h \rightarrow 0^{+}} \int_{t}^{t+h}\|\mathcal{T}(t+h-\xi) \mathcal{A} F(Y(\xi))\|_{\mathcal{X}_{s}} d \xi \\
& =0 .
\end{aligned}
$$

Hence, if $Y \in \mathcal{S}_{M}$, then $\Psi(Y) \in C\left([0, T] ; \mathcal{X}_{s}\right)$. On the other hand,

$$
\begin{aligned}
\left\|\Psi(Y(t))-\mathcal{T}(t) Y_{0}\right\|_{\mathcal{X}_{s}} & =\left\|\int_{0}^{t} \mathcal{T}(t-\xi) \mathcal{A} F(Y(\xi)) d \xi\right\|_{\mathcal{X}_{s}} \\
& \leq \int_{0}^{t} L\left(\|u\|_{s}, 0,\|\zeta\|_{s}\right)\|Y(\xi)\|_{\mathcal{X}_{s}} d \xi . \\
& \leq T \mathcal{K} L\left(M+\mathcal{K}\left\|Y_{0}\right\|_{\mathcal{X}_{s}}, 0, M+\mathcal{K}\left\|Y_{0}\right\|_{\mathcal{X}_{s}}\right)\left(M+\mathcal{K}\left\|Y_{0}\right\|_{\mathcal{X}_{s}}\right) .
\end{aligned}
$$

[Revista Integración 
Thus, choosing

$$
T_{1}=\frac{M}{\mathcal{K} L\left(M+\mathcal{K}\left\|Y_{0}\right\|_{\mathcal{X}_{s}}, 0, M+\mathcal{K}\left\|Y_{0}\right\|_{\mathcal{X}_{s}}\right)\left(M+\mathcal{K}\left\|Y_{0}\right\|_{\mathcal{X}_{s}}\right)},
$$

we obtain that $\Psi(Y(t)) \in \mathcal{S}_{M}$ provided $Y(t) \in \mathcal{S}_{M}$.

To see that there exists $T_{2}$ such that $\Psi$ is a contraction in $\mathcal{S}_{M}$ for $T<T_{2}$, let us observe that

$$
\begin{aligned}
\|\Psi(Y(t))-\Psi(\bar{Y}(t))\|_{\mathcal{X}_{s}} & =\left\|\int_{0}^{t} \mathcal{T}(t-\xi) \mathcal{A}[F(Y(\xi))-F(\bar{Y}(\xi))] d \xi\right\|_{\mathcal{X}_{s}}\|Y(\xi)-\bar{Y}(\xi)\|_{\mathcal{X}_{s}} . \\
& \leq \mathcal{K} T L\left(M+\mathcal{K}\left\|Y_{0}\right\|_{\mathcal{X}_{s}}, 0, M+\mathcal{K}\left\|Y_{0}\right\|_{\mathcal{X}_{s}}\right) \sup _{\xi \in[0, T]} \| Y(\xi)
\end{aligned}
$$

Thus, choosing

$$
T_{2}=\frac{1}{\mathcal{K} L\left(M+\mathcal{K}\left\|Y_{0}\right\|_{\mathcal{X}_{s}}, 0, M+\mathcal{K}\left\|Y_{0}\right\|_{\mathcal{X}_{s}}\right)},
$$

we obtain that $\Psi$ is a contraction on $\mathcal{S}_{M}$. Thus, with $T^{*}<\min \left\{T_{1}, T_{2}\right\}$ and by applying the Banach Fixed-Point Theorem on $\mathcal{S}_{M}$, we get the desired result.

Theorem 3.8. The solution obtained in Theorem 3.7 is unique and depends continuously on the initial condition $Y_{0}$.

Proof. Let $Y=\left(\begin{array}{l}\zeta \\ u\end{array}\right)$ and $\bar{Y}=\left(\begin{array}{l}\bar{\zeta} \\ \bar{u}\end{array}\right)$ be elements in $C\left([0, T] ; \mathcal{X}_{s}\right)$ solutions of the integral equation (11) with initial data $Y_{0}=\left(\begin{array}{c}\zeta_{0} \\ u_{0}\end{array}\right)$ and $\bar{Y}_{0}=\left(\begin{array}{c}\bar{\zeta}_{0} \\ \bar{u}_{0}\end{array}\right)$, respectively. Then,

$$
\begin{aligned}
\|Y(t)-\bar{Y}(t)\|_{\mathcal{X}_{s}} & =\left\|\mathcal{T}(t)\left(Y_{0}-\bar{Y}_{0}\right)+\int_{0}^{t} \mathcal{T}(t-\xi) \mathcal{A}[F(Y(\xi))-F(\bar{Y}(\xi))] d \xi\right\|_{\mathcal{X}_{s}} \\
& \leq \mathcal{K}\left\|Y_{0}-\bar{Y}_{0}\right\|_{\mathcal{X}_{s}}+\int_{0}^{t} \mathcal{K} L\left(\|u\|_{s},\|\bar{u}\|_{s},\|\zeta\|_{s}\right)\|Y(\xi)-\bar{Y}(\xi)\|_{\mathcal{X}_{s}} d \xi \\
& \leq \mathcal{K}\left(\left\|Y_{0}-\bar{Y}_{0}\right\|_{\mathcal{X}_{s}}+L\left(\|u\|_{s},\|\bar{u}\|_{s},\|\zeta\|_{s}\right) \int_{0}^{t}\|Y(\xi)-\bar{Y}(\xi)\|_{\mathcal{X}_{s}} d \xi\right) .
\end{aligned}
$$

Let $\bar{L}:=\sup _{t \in[0, T]} L\left(\|u(t)\|_{s},\|\bar{u}(t)\|_{s},\|\zeta(t)\|_{s}\right)$. The Gronwall inequality implies that

$$
\left.\|Y(t)-\bar{Y}(t)\|_{\mathcal{X}_{s}} \leq \mathcal{K}\left\|Y_{0}-\bar{Y}_{0}\right\|_{\mathcal{X}_{s}} e^{\bar{L} t}\right) \leq \mathcal{K}\left\|Y_{0}-\bar{Y}_{0}\right\|_{\mathcal{X}_{s}} e^{\bar{L} T},
$$

for all $t \in[0, T]$. Hence, we conclude the uniqueness and continuous dependence of the solutions on initial data.

\section{Numerical results}

In this section we will analyze the error of fully discrete numerical scheme for approximating the initial value problem for system (1) introduced by Muñoz in [12]. In this

Vol. 34, No. 1, 2016] 
numerical scheme, the spatial computational domain $[0, L]$ is discretized by $N \in 2 \mathbb{Z}$ equidistant points, with spacing $\Delta x=L / N$. Then, we approximate the unknowns $u$ and $\zeta$ with spatial period $L$ as truncated Fourier series in space with time-dependent coefficients:

$$
\begin{aligned}
& u(x, t)=\sum_{j=-N / 2+1}^{N / 2} \widehat{u}_{j}(t) e^{i w_{j} x}, \\
& \zeta(x, t)=\sum_{j=-N / 2+1}^{N / 2} \hat{\zeta}_{j}(t) e^{i w_{j} x},
\end{aligned}
$$

with

$$
w_{j}=\frac{2 \pi j}{L}, \quad j=-N / 2+1, \ldots 0, \ldots, N / 2 .
$$

The time-dependent coefficients $\widehat{u}_{j}(t)$ for $j=-N / 2+1, \ldots 0, \ldots, N / 2$ are calculated by means of the equation

$$
\widehat{u}_{j}(t)=\frac{1}{L} \int_{0}^{L} u(x, t) e^{-i w_{j} x} d x
$$

and similarly for $\hat{\zeta}_{j}(t)$.

Projecting equations (1) with respect to the orthonormal basis $\phi_{j}=L^{-1 / 2} e^{i w_{j} x}$ and the inner product

$$
\langle f, g\rangle=\int_{0}^{L} f(x) \overline{g(x)} d x
$$

we derive that

$$
\begin{aligned}
& \left\langle\zeta_{t}, \phi_{j}\right\rangle-\left\langle(u-\alpha \zeta u)_{x}, \phi_{j}\right\rangle=\frac{\epsilon^{2}}{6}\left\langle\zeta_{x x t}, \phi_{j}\right\rangle, \\
& \left\langle u_{t}, \phi_{j}\right\rangle+\frac{\alpha}{2}\left\langle\left(u^{2}\right)_{x}, \phi_{j}\right\rangle+\left(1-\rho_{r}\right)\left\langle\zeta_{x}, \phi_{j}\right\rangle=\rho_{r} \epsilon\left\langle\mathcal{H}\left(u_{x t}\right), \phi_{j}\right\rangle+\frac{\epsilon^{2}}{6}\left\langle u_{x x t}, \phi_{j}\right\rangle .
\end{aligned}
$$

Now substituting the Fourier expansions (16) into equations (17), using the orthogonal property of the basis $\phi_{j}$ and, integration by parts, we obtain

$$
\begin{aligned}
& \hat{\zeta}_{j}^{\prime}(t)-i w_{j} P_{j}\left[(u-\alpha \zeta u]=-\frac{\epsilon^{2}}{6} w_{j}^{2} \hat{\zeta}_{j}^{\prime},\right. \\
& \hat{u}_{j}^{\prime}(t)+\frac{i \alpha w_{j}}{2} P_{j}\left[u^{2}\right]+i w_{j}\left(1-\rho_{r}\right) \hat{\zeta}_{j}=\rho_{r} \epsilon \sum_{s} i w_{s} \widehat{u}_{s}^{\prime}(t)\left\langle\mathcal{H}\left(\phi_{s}\right), \phi_{j}\right\rangle-\frac{\epsilon^{2}}{6} w_{j}^{2} \hat{u}_{j}^{\prime},
\end{aligned}
$$

where $P_{j}[$.$] is the operator defined by$

$$
P_{j}[g]=\frac{1}{L} \int_{0}^{L} g(x) e^{-i w_{j} x} d x .
$$

Then, using the properties of the Hilbert Transform, system (18) reduces to

$$
\begin{aligned}
& \hat{\zeta}_{j}^{\prime}(t)-i w_{j} P_{j}\left[(u-\alpha \zeta u]=-\frac{\epsilon^{2}}{6} w_{j}^{2} \hat{\zeta}_{j}^{\prime},\right. \\
& \widehat{u}_{j}^{\prime}(t)+\frac{i \alpha w_{j}}{2} P_{j}\left[u^{2}\right]+i w_{j}\left(1-\rho_{r}\right) \hat{\zeta}_{j}=-\rho_{r} \epsilon\left|w_{j}\right| \widehat{u}_{j}^{\prime}-\frac{\epsilon^{2}}{6} w_{j}^{2} \hat{u}_{j}^{\prime} .
\end{aligned}
$$


Finally, we reach expressions for the Fourier coefficients of the unknowns $u$ and $\zeta$ :

$$
\begin{aligned}
\hat{\zeta}_{j}^{\prime} & =\frac{i w_{j}}{1+\frac{\epsilon^{2}}{6} w_{j}^{2}} P_{j}[(u-\alpha \zeta u)], \\
\widehat{u}_{j}^{\prime} & =\frac{i w_{j}\left(\rho_{r}-1\right) \hat{\zeta}_{j}}{1+\rho_{r} \epsilon\left|w_{j}\right|+\frac{\epsilon^{2}}{6} w_{j}^{2}}-\frac{i \alpha w_{j} P_{j}\left[u^{2}\right]}{2\left(1+\rho_{r} \epsilon\left|w_{j}\right|+\frac{\epsilon^{2}}{6} w_{j}^{2}\right)},
\end{aligned}
$$

subject to $\hat{\zeta}_{j}(0)=\hat{\zeta}_{0 j}, \hat{u}_{j}(0)=\hat{u}_{0 j}$. Equations $(20)$ can be considered as a system of ordinary differential equations for each frequency $w_{j}$, which we discretize numerically by the following second-order scheme:

$$
\begin{aligned}
\frac{\hat{\zeta}_{j}^{(n+1)}-\hat{\zeta}_{j}^{(n)}}{\Delta t}= & \frac{i w_{j}}{2}\left(\frac{\hat{u}_{j}^{(n+1)}+\hat{u}_{j}^{(n)}}{1+\frac{\epsilon^{2}}{6} w_{j}^{2}}\right)-\frac{3}{2}\left(\frac{i w_{j}}{1+\frac{\epsilon^{2}}{6} w_{j}^{2}}\right) P_{j}[\alpha \zeta u]^{(n)} \\
& +\frac{1}{2}\left(\frac{i w_{j}}{1+\frac{\epsilon^{2}}{6} w_{j}^{2}}\right) P_{j}[\alpha \zeta u]^{(n-1)}, \\
\frac{\widehat{u}_{j}^{(n+1)}-\widehat{u}_{j}^{(n)}}{\Delta t}= & \frac{i w_{j}\left(\rho_{r}-1\right)\left(\hat{\zeta}_{j}^{(n+1)}+\hat{\zeta}_{j}^{(n)}\right)}{2\left(1+\rho_{r} \epsilon\left|w_{j}\right|+\frac{\epsilon^{2}}{6} w_{j}^{2}\right)}-\frac{3 i \alpha w_{j} P_{j}\left[u^{2}\right]^{(n)}}{4\left(1+\rho_{r} \epsilon\left|w_{j}\right|+\frac{\epsilon^{2}}{6} w_{j}^{2}\right)} \\
& +\frac{i \alpha w_{j} P_{j}\left[u^{2}\right]^{(n-1)}}{4\left(1+\rho_{r} \epsilon\left|w_{j}\right|+\frac{\epsilon^{2}}{6} w_{j}^{2}\right)} .
\end{aligned}
$$

Here $\Delta t$ denotes the time step and $\widehat{u}_{j}^{(n)}, \hat{\zeta}_{j}^{(n)}$ denote the numerical approximations of the Fourier coefficients $\widehat{u}_{j}(t), \hat{\zeta}_{j}(t)$, respectively, at time $t=n \Delta t$. Also the notation $P_{j}[g]^{(n)}$ means the value of $P_{j}[g]$ when $g$ is evaluated at time $t=n \Delta t$.

\subsection{Description of the numerical experiments}

In this section, we wish to analyze the accuracy of the numerical scheme (21) described above in some numerical experiments.

Linear regime: First we solve system (1) with initial conditions

$$
\zeta(x, 0)=\cos \left(\frac{2 \pi x}{L}\right), u(x, 0)=-\cos \left(\frac{2 \pi x}{L}\right),
$$

and subject to spatial periodic conditions on an interval $[0, L]$. The parameters of the model are $\alpha=0$ (linear regime), $\epsilon=1, \rho_{r}=\rho_{2} / \rho_{1}=1.1, L=100$, and the numerical parameters are $N=2^{7}, \Delta t=0.01$. In Figure 2, we superimpose the exact solution computed in (7) in the linear case together with the output of the numerical scheme (21) evaluated at $t=100$. The difference between the profiles is about $1 e-8$ showing that the scheme reproduces very well the dispersive characteristics of model (1).

In Figure 3, we repeat the previous numerical experiment but using instead a weak level of dispersion $\epsilon=0.01$ and $\rho_{r}=3$. Other parameters and initial conditions are left unchanged. The result is presented in Figure 3. We see again a good agreement (of order $1 e-8)$ between the prediction of the numerical scheme and the exact solution given in (7). Neither numerical dispersion nor attenuation were observed in the simulations here presented and in many others performed for other values of the modelling parameters.

Vol. 34, No. 1, 2016] 

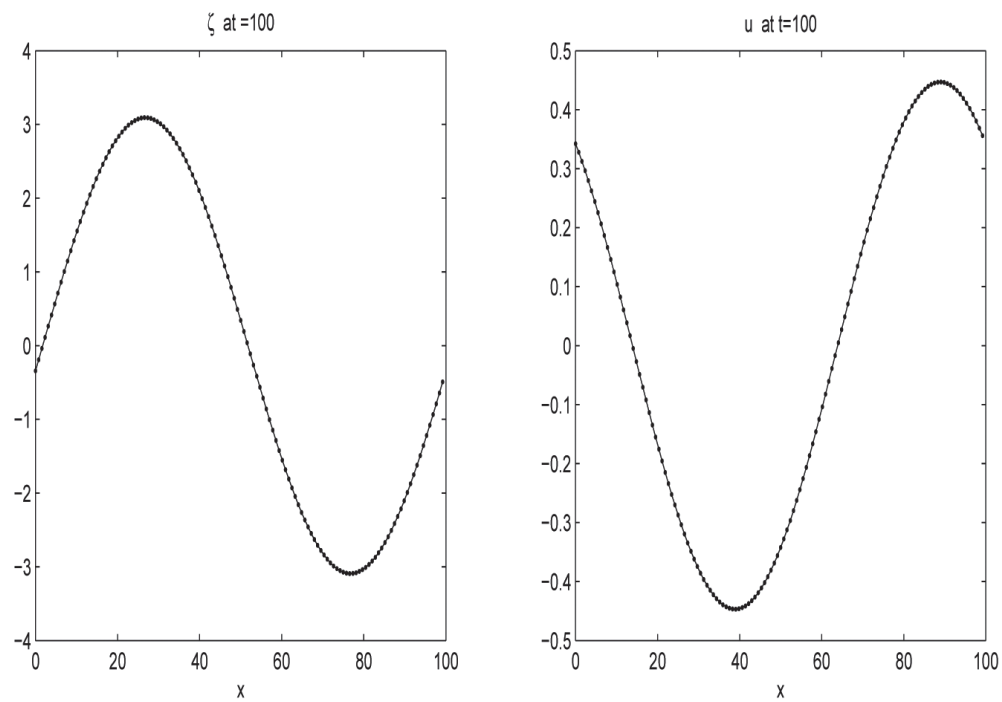

Figure 2. Solid line: Numerical solution of system $(1)$ in the linear case $(\alpha=0), \epsilon=1$ and $\rho_{r}=1.1$. Dotted line: exact solution (7).
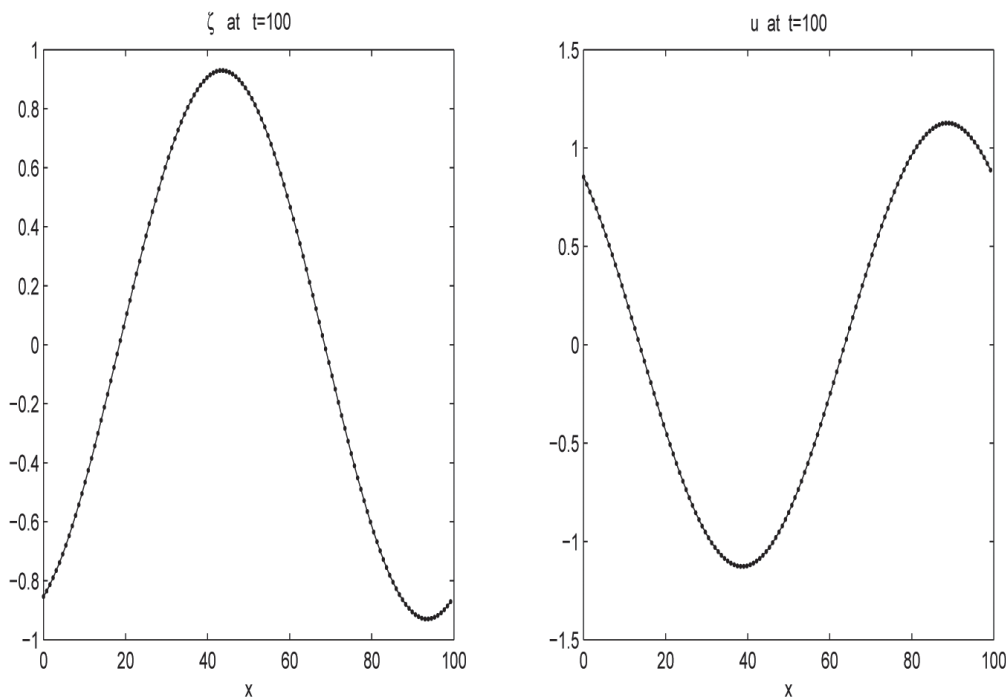

Figure 3. Solid line: Numerical solution of system (1) in the linear case $(\alpha=0), \epsilon=0.01$ and $\rho_{r}=3$. Dotted line: exact solution (7). 
Nonlinear regime: In the next numerical simulation, we conduct a computer experiment using an approximate periodic travelling wave with speed $c$ of system (1) in the form

$$
\zeta(x, t)=\zeta_{0}(x-c t), \quad u(x, t)=u_{0}(x-c t),
$$

with $\zeta_{0}, u_{0}$ being $L$-periodic functions showed in Figure 4, computed through the numerical scheme introduced in [14] with initial conditions

$$
\zeta_{0}(x)=10 \cos \left(\frac{2 \pi x}{L}\right), \quad u_{0}(x)=10 \cos \left(\frac{2 \pi x}{L}\right),
$$

and $L=4, c=1.5, \alpha=\epsilon=0.1, \rho_{r}=2$. We run the numerical solver (21) using the profiles in Figure 4 as initial conditions and $\Delta t=5 e-4, N=2^{8}, L=4$. In Figure 5, the resulting profiles are displayed at $t=20$. We observe that the two profiles coincide with accuracy of roughly $3 e-4$, and the travelling wave propagates with the expected speed without changing its shape.
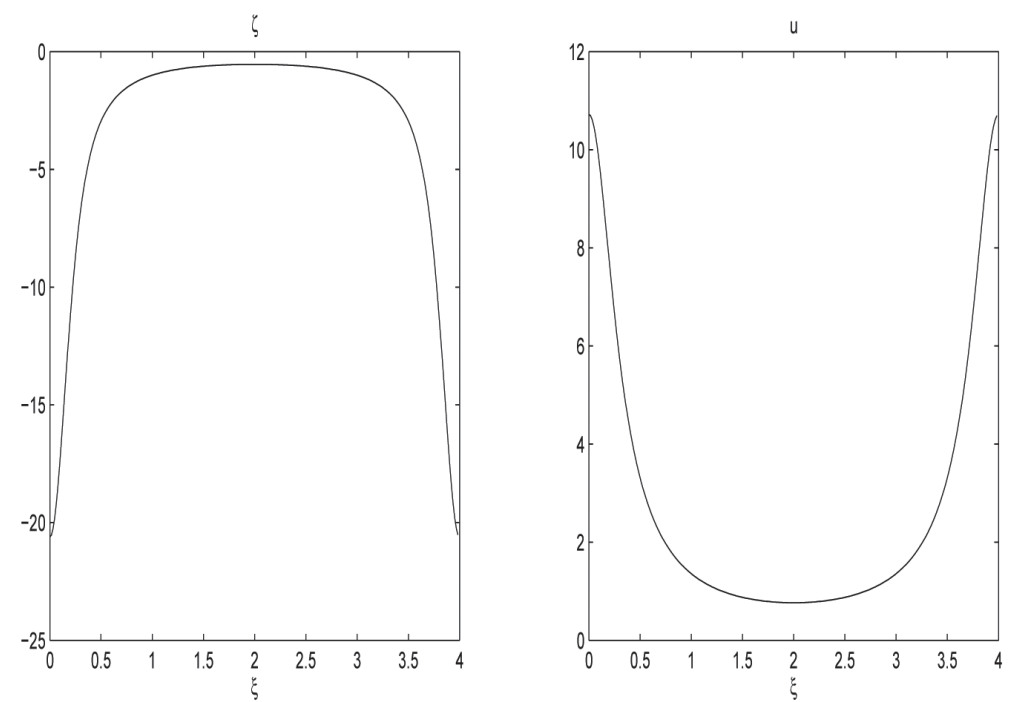

Figure 4. Approximate travelling wave of system (1) computed using the numerical method in [14] after 10 Newton's iterations. Period $L=4$, wave speed $c=1.5, \rho_{r}=2, \alpha=\epsilon=0.1, N=2^{8}$ FFT points in space.

\subsection{Order in space of the Fourier method}

We wish to analyze the order of convergence in space of the fully discrete numerical method (21). In the experiment displayed in Figure 6, we fix a small time step $\Delta t=1 e-4$ and increase gradually the number of FFT points in space. We use the approximate periodic travelling wave in Figure 4 with the same parameters. We start with $N=2^{5}$ and increase by 2 until we get $N=2^{8}$. In each simulation, we run the numerical solver (21) within the time interval $[0,1]$, for every value of $N$. From Figure 6 , we can see

Vol. 34, No. 1, 2016] 

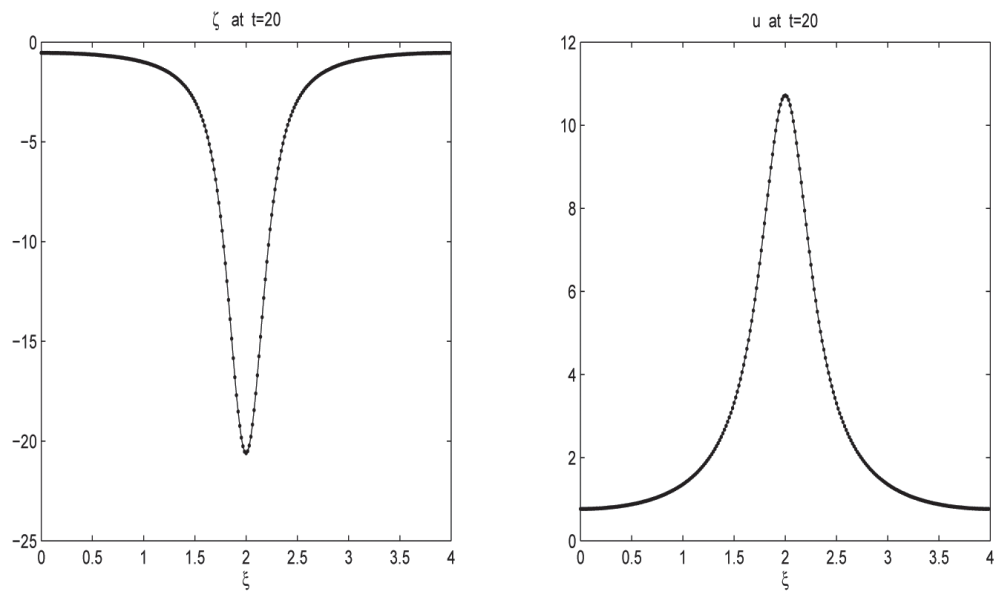

Figure 5. Solid line: Numerical solution with method (21) of system (1) for $\alpha=0.1$ and $\epsilon=0.1, \rho_{r}=2$. Dotted line: Approximate periodic travelling wave computed in [14] at $t=20$ (5 times its period).

that the error in space of scheme (21) decreases very rapidly approximately as $N^{-7.8}$, in accordance with the spectral accuracy of the semidiscrete approximation established by Muñoz in [12].

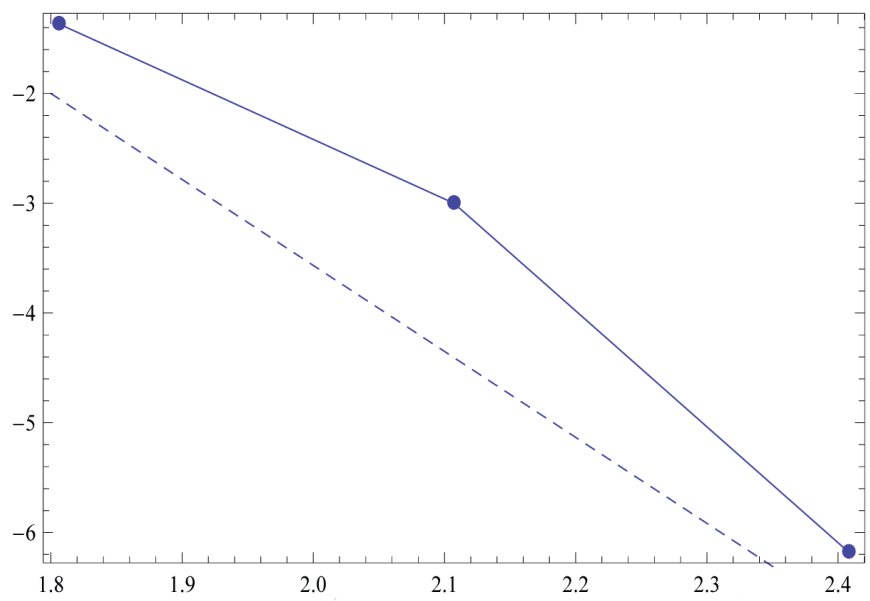

Figure 6. Plot of the decimal logarithm of the maximum error against $\log _{10} N$. The time step is fixed at $\Delta t=1 e-4$. We see that the plot is approximately a line (see dashed line) with slope -7.8 .

\subsection{Order in time of the Fourier method}

Now, we want to check numerically the order in time for the numerical scheme (21). We use the same periodic travelling wave of system (1) as in the previous experiment. We 
choose $N=2^{18}(\Delta x=L / N \approx 1.5 e-4)$, the error in space does not dominate the total error. We start with $\Delta t=1 / 2$ and decreasing the time step by $1 / 2$ until $\Delta t=1 / 2^{6}$. In each computer simulation, we run the numerical solver (21) within the time interval $[0,1]$, for every value of $\Delta t$. In Figure 7 , we can see that the error of scheme (21) is approximately of order 2 in time.

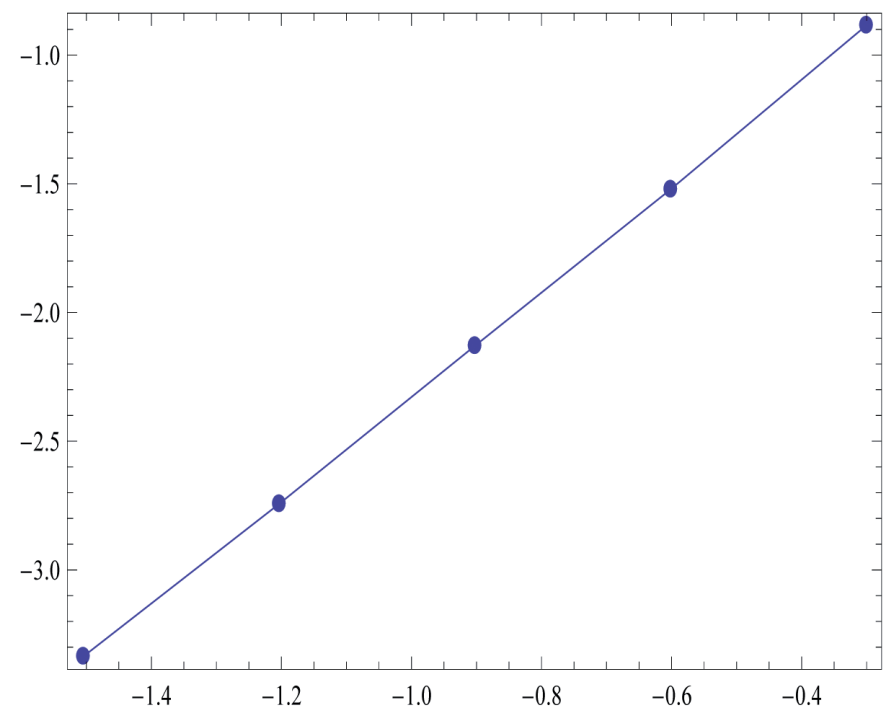

Figure 7. Plot of the decimal logarithm of the maximum error against $\log _{10} N$. The number of points in space is fixed at $N=2^{18}$. We see that the plot is approximately a line with slope 2 .

\section{Acknowledgments}

This work was supported by Universidad del Valle, Calle 13 No. 100-00, Cali, Colombia, under the internal research project C.I. 71020, and Colciencias. The authors would like to thank the referees for their valuable comments which helped to improve the manuscript.

\section{References}

[1] Benjamin T.B., Bona J.L. and Bose D.K., "Solitary-wave solutions of nonlinear problems", Philos. Trans. Roy. Soc. London Ser. A 331 (1990), No. 1617, 195-244.

[2] Bona J.L., Lannes D. and Saut J.C, "Asymptotic models for internal waves", J. Math. Pures Appl. (9) 89 (2008), No. 6, 538-566.

[3] Butzer P.L. and Nessel R.J., Fourier analysis and approximation. Volume 1: Onedimensional theory, Pure and Applied Mathematics, Vol. 40, Academic Press, New York-London, 1971. 
[4] Chen H., "Existence of periodic travelling-wave solutions of nonlinear, dispersive wave equations", Nonlinearity 17 (2004), No. 6, 2041-2056.

[5] Choi W. and Camassa R., "Fully nonlinear internal waves in a two-fluid system", $J$. Fluid. Mech. 396 (1999), 1-36.

[6] Choi W. and Camassa R., "Long internal waves of finite amplitude", Phys. Rev. Lett. 77 (1996), No. 9, 1759-1762.

[7] Choi W. and Camassa R., "Weakly nonlinear internal waves in a two-fluid system", J. Fluid Mech. 313 (1996), 83-103.

[8] Duoandikoetxea J., Fourier analysis, Graduate Studies in Mathematics 29, American Mathematical Society, Providence, RI, 2001.

[9] Granas A., "The Leray-Shauder index and the fixed point theory for arbitrary ANRs", Bull. Soc. Math. France 100 (1972), 209-228.

[10] Krasnosel'skii M.A., Positive solutions of operator equations, P. Noordhoff Ltd. Groningen, 1964.

[11] Krasnosel'skii M.A., Topological methods in the theory of nonlinear integral equations, A Pergamon Press Book, The Macmillan Co., New York, 1964.

[12] Muñoz Grajales J.C., "Existence and numerical approximation of solutions of an improved internal wave model", Math. Model. Anal. 19 (2014), No. 3, 309-333.

[13] Pazy A., Semigroups of linear operators and application to partial differential equations, Applied Mathematical Sciences 44, Springer-Verlag, New York, 1983.

[14] Pipicano F.A. and Muñoz Grajales J.C., "Existence of periodic travelling wave solutions for a regularized Benjamin-Ono system", J. Differential Equations 259 (2015), No. $12,7503-7528$.

[15] Quintero J. and Muñoz Grajales J.C., "Solitary waves for an internal wave model", submitted to Discrete Contin. Dyn. Syst., 2015.

[16] Roumégoux D., "A symplectic non-squeezing theorem for BBM equation", Dyn. Partial Differ. Equ. 7 (2010), No. 4, 289-305. 